\title{
Effect of silicone gel versus Contractubex or corticosteroid phonophoresis for post-burn hypertrophic scars: a single-blind randomized controlled trial
}

\author{
DOI: https://doi.org/10.5114/pq.2019.83054
}

\author{
Ereny S. Wahba', Hamada Ahmed Hamada ${ }^{2}$, Ayman El Khatib ${ }^{3}$ \\ ${ }^{1}$ Department of Physical Therapy for Surgery, Faculty of Physical Therapy, Cairo University, Cairo, Egypt \\ 2 Department of Biomechanics, Faculty of Physical Therapy, Cairo University, Cairo, Egypt \\ ${ }^{3}$ Physical Therapy Department, Faculty of Health Sciences, Beirut Arab University, Beirut, Lebanon
}

\section{Abstract}

Introduction. Hypertrophic scars and keloids are physically and mentally disturbing, and can cause pain and itching. Various treatment is utilized to diminish or counteract scarring. The purpose of this study was to compare the effect of silicone gel, Contractubex gel, and corticosteroid phonophoresis for post-burn hypertrophic scars.

Methods. A prospective, single-blind, randomized, controlled study with pretest-posttest design was performed; 45 patients aged 20-45 years with hypertrophic scars, $2-4$ months post thermal burn were involved. They were randomly assigned to 3 groups: group A ( 15 patients, 8 males and 7 females) received phonophoresis with silicone gel, group B ( 15 patients, 8 males and 7 females) received phonophoresis with Contractubex gel, and group C (15 patients, 9 males and 6 females) received corticosteroid phonophoresis. All treatment interventions were applied at the frequency of 3 sessions/week for 24 weeks. The participants were recruited from the Kasr El-Ani hospital outpatient clinic to be treated in the outpatient clinic, Faculty of Physical Therapy, Cairo University.

Results. Outcome measures were taken with the modified Vancouver Scar Scale (VSS) before the treatment, and 12 and 24 weeks after the intervention. The comparison among the 3 groups revealed a highly significant decrease in modified VSS total score in group A compared with group B and group C $(p<0.01)$, as well as in group B compared with group $C(p<0.001)$. Conclusions. Silicone gel phonophoresis is a more effective method for post-burn hypertrophic scar management than Contractubex gel phonophoresis or corticosteroid phonophoresis.

Key words: hypertrophic scar, silicone gel, Contractubex gel, corticosteroid, phonophoresis

\section{Introduction}

Hypertrophic scars are severe problems after thermal injuries. Scar contractures develop and extend to the underlying connective tissue and then muscles, leading to a reduction in joint range of motion and affecting the day-to-day activities [1].

Hypertrophic scars are commonly raised, erythematous, hard, inflexible, and contracted. They are composed of an extracellular matrix, predominantly collagen. Mature elastin fibres do not exist within the extracellular scar matrix [2].

The incidence of hypertrophic scars resulting from burns stays doubtful, with estimates of $26-75 \%$, depending on age, ethnicity, and if recovery was spontaneous or took place via surgical approach [3]. Apart from various individual troubles, patients often complain of itching, redness, and hard nodular scar tissue, regularly with extraordinary sensations [4].

The treatment of hypertrophic scars consists of compression therapy, topical/intralesional corticosteroid use, excisional surgical treatment, radiation, cryotherapy, laser therapy, silicone gel sheeting; these methods may be used alone or in combinations, as treatment plans [5]. Conservative treatments had been preferred within scientific settings to keep in the progression of scar and contracture with the non-invasive and easy-operation management [6].

The advantages of silicone are associated with occlusion and hydration of scars. About 1-2 weeks after tissue injury, an increase of transepidermal water loss occurs, leading to stratum corneum dehydration. Consequently, interleukin 1 activation stimulates proliferation of keratinocytes, together with restoring barrier function and preventing dehydration. Interleukin 1 signals the fibroblasts in the dermis to increase the formation of collagen. The utilization of silicone upon scars restores the barrier function of the stratum corneum, lowering transepidermal water loss, interrupting the development of keratinocytes, and terminating the growth of fibroblasts in the dermis, therefore normalizing collagen formation [7]. The production of basic fibroblast growth factor may be accelerated with the aid of silicone products [8]. Elevated basic fibroblast growth factor levels in fibroblasts lead to reduced collagen production.

Contractubex gel (10\% aqueous onion extract, $50 \mathrm{U}$ heparin per gram of gel, $1 \%$ allantoin) is a commonly used ointment in routine outpatient practice claimed to be effective in the treatment of scars [9]. Onion extract gel decreased scar width, lowered scar erythema [10], reduced pruritus, and generally improved scar appearance [11]. Contractubex gel is effective in preventing the development of pathologic signs in freshly healing scars (6-12 days old). On the other hand, well-formed hypertrophic scars respond excellently to products containing enzymes that display fibrinolytic activity [12].

Corticosteroid injections (e.g., triamcinolone acetonide) may be used to treat hypertrophic scars and keloids as mono- 
therapy or in combination with other therapies. The response rate with this treatment equals $50-100 \%$, and the recurrence rate is $9-50 \%$ [13]. Corticosteroids suppress recovery by way of three different mechanisms. Firstly, inflammation is suppressed by prevention of leukocyte and monocyte migration and phagocytosis. Secondly, corticosteroids are strong vasoconstrictors, so they limit the transport of oxygen and nutrients to the wound bed. Thirdly, the antimitotic effect inhibits keratinocytes and fibroblasts, thereby slowing epithelialization and new collagen formation. Corticosteroid injections seem to limit collagen formation by lowering the inflammatory process in the wound, reducing fibroblast proliferation, and causing hypoxia [14].

Ultrasound (phonophoresis) is a method for improving the skin permeation of drugs. It combines ultrasound therapy with topical drug therapy to gain therapeutic drug concentrations at selected sites within the skin. In this technique, the drug is combined with a coupling agent, commonly a gel [15].

Application of low-frequency ultrasound (20-100 kHz) enhances skin permeability more efficiently than high-frequency ultrasound (1-16 MHz). The mechanism of transdermal permeation involves a breakdown of the stratum corneum lipids, therefore allowing the drug to pass via the skin. A corresponding reduction in skin resistance was observed after cavitation, microstreaming, and heat generation [16]. Phonophoresis via ultrasound has a number of advantages; the risk of burning the skin is low, and it is unnecessary to ionize the drug.

Previous studies have focused only on the efficacy of silicon alone, Contractubex, or corticosteroids, but no research has been conducted to compare those different methods and apply ultrasound waves to improve the skin absorption of the medications $[15,17,18]$.

The purpose of this study was to compare the effectiveness of topical silicone gel phonophoresis, Contractubex phonophoresis, and corticosteroid phonophoresis for postburn hypertrophic scars.

\section{Subjects and methods}

\section{Study design}

A prospective, single-blind, randomized, controlled study with pretest-posttest design and 3 parallel groups was performed between June 2017 and January 2018. The Consolidated Standards of Reporting Trials were followed. The study was registered in ClinicalTrial.gov under the identifier NCT03777891.

\section{Participants}

A convenient sample of 45 patients ( 25 males and 20 females) were recruited from the Kasr El-Ani hospital outpatient clinic to be treated in the outpatient clinic, Faculty of Physical Therapy, Cairo University. They were enrolled and assessed for their eligibility to participate in the study. Included were patients with hypertrophic scars 2-4 months after a deep second-degree thermal burn (direct flame or scald), aged 20-45 years, free from any other illness that might affect the results, such as skin disease, diabetes, mental disorders, peripheral vascular diseases, acute viral diseases, open wound, electrical or chemical burns.

\section{Randomization}

The participants were informed about the nature, purpose, and benefits of the study, their right to refuse or withdraw at any time, and the confidentiality of any obtained information. Anonymity was assured through coding of all data. The subjects with hypertrophic post-burn scars were randomly assigned into 3 groups (A, B, and $C$ ) by a blinded and independent research assistant who opened sealed envelopes that contained a computer-generated randomization card. No subjects dropped out of the study after randomization.

\section{Interventions}

Patients randomly assigned to group A received silicone gel phonophoresis: the gel (Strataderm) was applied to the scar and then the ultrasound was implemented by the therapist. The ultrasound parameters were as follows: frequency: $1 \mathrm{MHz}$, intensity: $0.5 \mathrm{~W} / \mathrm{cm}^{2}$, treatment time: 5 minutes. The therapeutic ultrasound device was Nonius Sonopuls 590 S.NO.03-202 type 14663.900 (Enraf, Holland).

Group B received Contractubex phonophoresis: Contractubex (Merz Pharma, Frankfurt, Germany) was applied to the scar and then the ultrasound was implemented by the therapist. The ultrasound parameters were as follows: frequency: $1 \mathrm{MHz}$, intensity: $0.5 \mathrm{~W} / \mathrm{cm}^{2}$, treatment time: 5 minutes.

Group $\mathrm{C}$ was treated with corticosteroid phonophoresis: a thin film of coupling medium (gel) was put on the hypertrophic scar and a sufficient quantity of triamcinolone was applied with a syringe over the whole scar; then the ultrasound was implemented by the therapist. The ultrasound parameters were as follows: frequency: $1 \mathrm{MHz}$, intensity: $0.5 \mathrm{~W} / \mathrm{cm}^{2}$, treatment time: 5 minutes.

All groups received 72 sessions of treatment, 3 times per week for 24 weeks.

\section{Outcome measures}

Scar assessment was performed with the use of the modified Vancouver Scar Scale (VSS) [19]. It consists in a numerical evaluation of 4 skin characteristics, where 0 represents normal skin. The characteristics include height (range: 0-3), pliability (range: 0-5), vascularity (range: $0-3$ ), and pigmentation (range: $0-3$ ). The assessment was carried out before treatment, 3 months ( 12 weeks) after treatment, and 6 months (24 weeks) after treatment. The outcome was determined after 6 months of follow-up in terms of complete or incomplete recovery.

\section{Statistical analysis and sample size calculation}

The sample size was determined by the availability of participants that could be recruited within the fixed time of data collection. Statistical analysis was conducted with the SPSS for Windows software, version 23 (SPSS, Inc., Chicago, USA). The test involved 1 dependent variable (VSS) and 2 independent variables; the between-subject factor had 3 levels (group A received phonophoresis with silicone gel, group B received phonophoresis with Contractubex gel, and group $\mathrm{C}$ received corticosteroid phonophoresis) and the within-subject factor had 3 levels (measuring periods: before treatment, 12 weeks after treatment, 24 weeks after treatment). Accordingly, $3 \times 3$ mixed design ANOVA was used to compare the VSS results of different measuring periods in 3 different groups. The preliminary assumption checking revealed that data were normally distributed, as 
assessed by a Normal Q-Q Plot. The examination of studentized residuals for values greater than \pm 3 indicated that there were no outliers. The Levene's test of homogeneity of variances and Box's $M$ test determined homogeneity of variances $(p>0.05)$ and covariances $(p>0.05)$, respectively. Mauchly's test of sphericity indicated that the assumption of sphericity was not violated for the two-way interaction $\left(\chi^{2}(2)=0.678, p=0.712\right)$. The alpha level was set at 0.05 .

\section{Ethical approval}

The research related to human use has been complied with all the relevant national regulations and institutional policies, has followed the tenets of the Declaration of Helsinki, and has been approved by the Ethics Committee of the Faculty ofPhysicalTherapy, CairoUniversity(P.T.REC/012/001870).

\section{Informed consent}

Informed consent has been obtained from all individuals included in this study.

\section{Results}

The total of 60 patients with hypertrophic scars $2-4$ months after a post-thermal burn were eligible for inclusion, and 45 were randomized for the study intervention (Figure 1). Baseline demographic characteristics of the safety population are presented in Table 1.

Group A received phonophoresis with silicone gel, group B received phonophoresis with Contractubex gel, and group $C$ received corticosteroid phonophoresis. As indicated by the one-way ANOVA test, there were no statistically significant differences $(p>0.05)$ among the 3 groups concerning age. In addition, the chi-square test revealed no significant differences among the 3 groups in sex distribution $(p>0.05)$.

The statistical analysis with $3 \times 3$ mixed design ANOVA involved 45 patients assigned to 3 equal groups. It revealed that there were significant within-subject $(F=550.467, p=$ $0.0001)$, treatment ${ }^{\star}$ time $(F=21.081, p=0.0001)$, and between-subject ( $F=22.277, p=0.0001)$ effects. Table 2 presents descriptive statistics and the results of multiple pairwise comparison tests (post-hoc tests) for VSS in different measuring periods in the 3 groups. The multiple pairwise comparison tests showed that there was a significant reduction $(p<0.05) 24$ weeks after treatment as compared with before treatment and 12 weeks after treatment. In addition, a significant reduction $(p<0.05)$ was observed 12 weeks after treatment as compared with before treatment in the 3 groups. Regarding between-subject effects, multiple pairwise comparisons proved a significant reduction $(p<0.05)$ in VSS 12 weeks after treatment and 24 weeks after treatment in group A as compared with groups B and C. There was also a significant reduction $(p<0.05)$ in group $B$ as compared with group C 12 weeks after treatment and 24 weeks after treatment.

No adverse events occurred during the study.

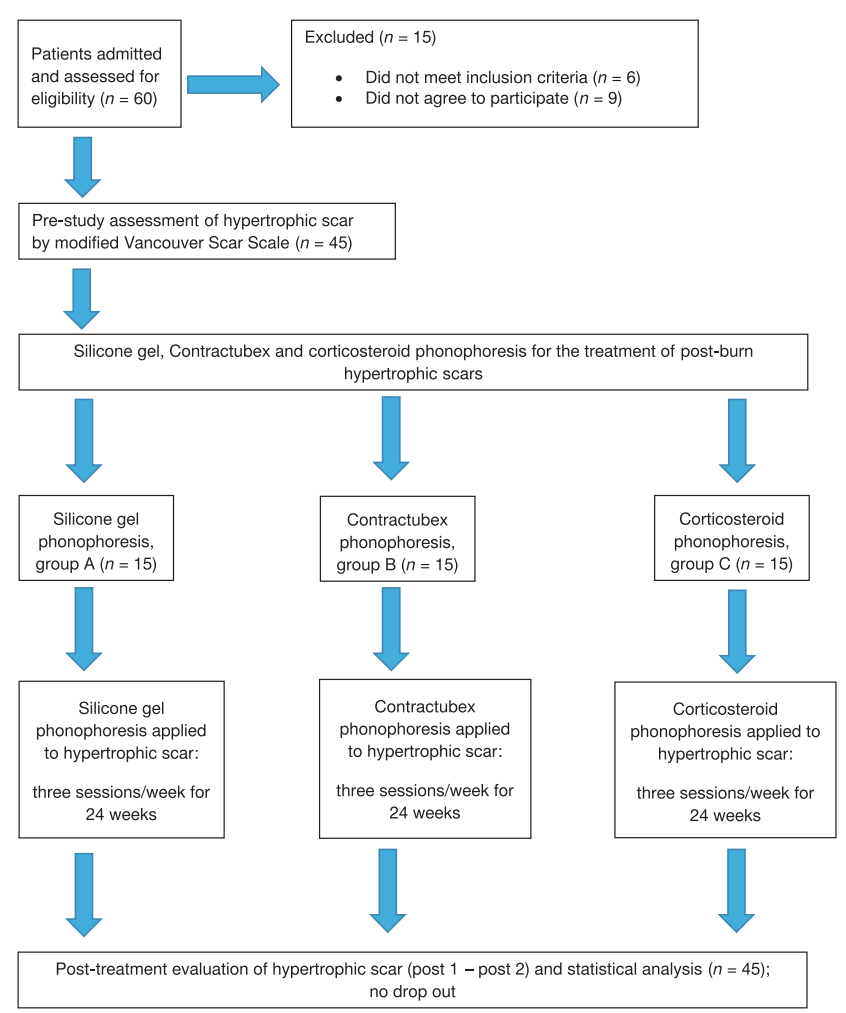

Figure 1. Flow chart of the study

\section{Discussion}

The present study was designed to compare the efficacy of silicone gel phonophoresis (group A), Contractubex gel phonophoresis (group B), and corticosteroid phonophoresis (group C) for post-burn hypertrophic scars. The assessment was performed with the use of modified VSS among 45 patients suffering from post-burn hypertrophic scars.

The results revealed that there was no significant difference in any item of modified VSS between the groups before treatment, while a significant decrease was observed in the total score of modified VSS in group A 12 weeks after treatment and 24 weeks after treatment compared with group $B$ and group $C$. In addition, there was a significant decrease in the total score of modified VSS in group B 12 weeks after treatment and 24 weeks after treatment compared with group $\mathrm{C}$. That could prove that silicone gel phonophoresis and Contractubex gel phonophoresis are more effective methods in the treatment of hypertrophic scars than corticosteroid phonophoresis, with silicone gel phonophoresis being superior to Contractubex phonophoresis and corticosteroid phonophoresis.

The study was significant for two reasons. Firstly, it was conducted for determination and comparison of the effectiveness of three topical agents (silicone gel, Contractubex gel, and corticosteroid) by measuring the decrease in the scar tissue thickness. Secondly, the study was performed to evalu-

Table 1. Demographic characteristics of the study groups

\begin{tabular}{|l|c|c|c|c|}
\cline { 2 - 5 } \multicolumn{1}{c|}{} & \begin{tabular}{c} 
Group A \\
\multicolumn{1}{c|}{}
\end{tabular} & $\begin{array}{c}\text { Group B } \\
\text { (mean } \pm S D)\end{array}$ & $\begin{array}{c}\text { Group C } \\
(\text { mean } \pm S D)\end{array}$ & $p$ value \\
\hline Age (years) & $32.66 \pm 6.83$ & $31.53 \pm 5.6$ & $33.13 \pm 6.47$ & 0.77 \\
\hline Males/females & $8 / 7$ & $8 / 7$ & $9 / 6$ & 0.91 \\
\hline
\end{tabular}


Table 2. Descriptive statistics and $3 \times 3$ mixed design ANOVA results for Vancouver Scar Scale for different measuring periods in the 3 study groups

\begin{tabular}{|l|c|c|c|}
\hline \multirow{2}{*}{ Group } & \multicolumn{3}{|c|}{ Vancouver Scar Scale } \\
\cline { 2 - 4 } & $\begin{array}{c}\text { Before treatment } \\
(\text { mean } \pm S D)\end{array}$ & $\begin{array}{c}12 \text { weeks after treatment } \\
(\text { mean } \pm S D)\end{array}$ & $\begin{array}{c}24 \text { weeks after treatment } \\
(\text { mean } \pm S D)\end{array}$ \\
\hline A & $10.26 \pm 1.22$ & $7.13 \pm 1.12$ & $3.86 \pm 0.91$ \\
\hline B & $10.46 \pm 0.99$ & $8.53 \pm 0.91$ & $6.06 \pm 1.09$ \\
\hline C & $10.73 \pm 1.27$ & $9.8 \pm 1.08$ & $8 \pm 1.3$ \\
\hline
\end{tabular}

Multiple pairwise comparisons (post-hoc tests) for the Vancouver Scar Scale among different measuring periods in different groups

\begin{tabular}{|l|c|c|c|}
\hline \multirow{2}{*}{ Group } & \multicolumn{2}{|c|}{$p$ value } \\
\cline { 2 - 4 } & $\begin{array}{c}\text { Before treatment vs. } \\
12 \text { weeks after treatment }\end{array}$ & $\begin{array}{c}\text { Before treatment vs. } \\
24 \text { weeks after treatment }\end{array}$ & $\begin{array}{c}12 \text { weeks after treatment vs. } \\
\text { 24 weeks after treatment }\end{array}$ \\
\hline A & $0.0001^{*}$ & $0.0001^{*}$ & $0.0001^{*}$ \\
\hline B & $0.0001^{*}$ & $0.0001^{*}$ & $0.0001^{*}$ \\
\hline C & $0.0001^{*}$ & $0.0001^{*}$ & $0.0001^{*}$ \\
\hline
\end{tabular}

Multiple pairwise comparisons (post-hoc tests) for the Vancouver Scar Scale among the 3 groups in different measuring periods

\begin{tabular}{|l|c|c|c|}
\hline \multirow{2}{*}{ Measuring period } & \multicolumn{2}{|c|}{$p$ value } & Group B vs. group C \\
\cline { 2 - 4 } & Group A vs. group B & Group A vs. group C & 0.99 \\
\hline Before treatment & 0.99 & 0.844 & $0.006^{*}$ \\
\hline 12 weeks after treatment & $0.002^{*}$ & $0.0001^{*}$ & $0.0001^{*}$ \\
\hline
\end{tabular}

* Significant at alpha level $<0.05$

ate the effectiveness of ultrasound and a topical agent on burn scars with regard not only to pruritus, erythema, and pigmentation, but also to the reduction of scar tissue thickness.

The results are in line with those of a study that found silicone gel superior to onion extract for the prevention of hypertrophic scars in the rabbit ear [20]. The results also agree with a study that confirmed that silicone gel significantly improved the roughness of the scars and that patients experienced significantly less itching during 6 months of treatment [17]. The findings of the current study were supported by Karagoz et al. [18], who showed that silicone gel was as effective as silicone sheeting at improving scars as assessed with VSS in a 6-month study of 45 post-burn hypertrophic scars. Both silicone products turned out significantly more effective at improving these scars than Contractubex, a topical onion extract containing heparin and allantoin. In addition, the current findings are in line with the observation that onion extract gel resulted in fewer hypertrophic scars than placebo in paediatric median sternotomies [21].

Chanprapaph et al. [22] showed that onion extract improved scar height and itchiness but not erythema, pliability, or general appearance, as compared with placebo. A quick use of onion extract gel in surgical scars resulted in a decrease in the scar height and symptoms; however, there was no statistically significant difference in the scar redness, pliability, or overall cosmetic appearance.

Ho et al. [23] found that onion extract, heparin, and allantoin gel was effective, safe, and easy to use for the counteraction of scarring among 120 Chinese patients undergoing laser removal of tattoos. The researchers observed that the topical gel preparation decreased the risk of scarring significantly, by $11.5-23.5 \%$. Nevertheless, we have observed that many patients who use a topical preparation containing onion extract, heparin, and allantoin gel or another onion extract gel do not notice any significant improvement in their hypertrophic scars.

Beuth et al. [9] compared hypertrophic scars treated with Contractubex for 28 days and those after 1 intralesional corticosteroid application. Contractubex allowed for a significantly shorter period of scar normalization (erythema, pruritus, and consistency) than that in the corticosteroid group. Additionally, Contractubex treatment was associated with significantly fewer adverse events (e.g., cutaneous atrophy of scar and surrounding skin tissue) than intralesional corticosteroid injections.

Juckett and Hartman-Adams [13] observed a 50-100\% response rate with corticosteroid triamcinolone acetonide, and a $9-50 \%$ recurrence rate. They found that the local adverse events included skin and subcutaneous tissue atrophy, capillary dilatation, and hypopigmentation. In addition, Koc et al. [25] noted 50-100\% response rates with corticosteroid triamcinolone acetonide, and a recurrence rate of 9-50\%. Despite the small number of randomized studies, there is broad consensus that injected triamcinolone acetonide is effective; it constitutes first-line therapy for keloids and second-line therapy (if other, easier treatments have not been efficacious) for hypertrophic scars [24].

\section{Limitations}

Although silicone gel is the most effective agent for hypertrophic scars treatment, it has some limitations as it is very expensive; therefore, the findings of the study may be limited by cost-effectiveness from a health service perspective. In addition, the study lacked a follow-up of the post-burn hypertrophic scars among the analysed groups for several months after a rehabilitation program to evaluate the long-lasting effect. 


\section{Conclusions}

Silicone gel phonophoresis is a more effective method for the treatment of post-burn hypertrophic scars than Contractubex gel phonophoresis or corticosteroid phonophoresis. Pigmentation, vascularity, pliability, and height are reduced significantly with silicone gel phonophoresis in comparison with the other methods.

\section{Disclosure statement}

No author has any financial interest or received any financial benefit from this research.

\section{Conflict of interest}

The authors state no conflict of interest.

\section{References}

1. Dewey WS, Richard RL, Parry IS. Positioning, splinting, and contracture management. Phys Med Rehabil Clin N Am. 2011;22(2):229-247; doi: 10.1016/j.pmr.2011.02.001.

2. Sidgwick GP, Bayat A. Extracellular matrix molecules implicated in hypertrophic and keloid scarring. J Eur Acad Dermatol Venereol. 2012;26(2):141-152; doi: 10.1111 /j.1468-3083.2011.04200.x.

3. Gangemi EN, Gregori D, Berchialla P, Zingarelli E, Cairo $\mathrm{M}$, Bollero $\mathrm{D}$, et al. Epidemiology and risk factors for pathologic scarring after burn wounds. Arch Facial Plast Surg. 2008;10(2):93-102; doi: 10.1001/archfaci.10.2.93.

4. Schneider JC, Holvanahalli R, Helm P, Goldstein R, Kowalske K. Contractures in burn injury: defining the problem. J Burn Care Res. 2006;27(4):508-514; doi: 10.1097/ 01.BCR.0000225994.75744.9D.

5. Tsao SS, Dover JS, Arndt KA, Kaminer MS. Scar management: keloid, hypertrophic, atrophic, and acne scars. Semin Cutan Med Surg. 2002;21(1):46-75; doi: 10.1016/ S1085-5629(02)80719-2.

6. Anthonissen M, Daly D, Janssens T, Van den Kerckhove $\mathrm{E}$. The effects of conservative treatments on burn scars: a systematic review. Burns. 2016;42(3):508-518; doi: 10.1016/j.burns.2015.12.006.

7. Mustoe TA. Evolution of silicone therapy and mechanism of action in scar management. Aesthetic Plast Surg. 2008;32(1):82-92; doi: 10.1007/s00266-007-9030-9.

8. Hanasono MM, Lum J, Carroll LA, Mikulec AA, Koch RJ. The effect of silicone gel on basic fibroblast growth factor levels in fibroblast cell culture. Arch Facial Plast Surg. 2004;6(2):88-93; doi: 10.1001/archfaci.6.2.88.

9. Beuth J, Hunzelmann N, Van Leendert R, Basten R, Noehle M, Schenider B. Safety and efficacy of local administration of Contractubex ${ }^{\circledR}$ to hypertrophic scars in comparison to corticosteroid treatment. Results of a multicenter, comparative epidemiological cohort study in Germany. In Vivo. 2006;20(2):277-283.

10. Campanati A, Savelli A, Sandroni L, Marconi B, Giuliano A, Giuliodori K, et al. Effect of Allium cepa-allantoinpentaglycan gel on skin hypertrophic scars: clinical and video-capillaroscopic results of an open-label, controlled, nonrandomized clinical trial. Dermatol Surg. 2010;36(9): 1439-1444; doi: 10.1111/j.1524-4725.2010.01654.x.

11. Ocampo-Candiani J, Vázquez-Martínez OT, Iglesias Benavides JL, Buske K, Lehn A, Acker C. The prophylactic use of a topical scar gel containing extract of Allium cepae, allantoin, and heparin improves symptoms and appearance of cesarean-section scars compared with untreated scars. J Drugs Dermatol. 2014;13(2):176-182.
12. Gerasimenko MI, lusova ZI, Zenger VG, Kazantseva IA A differentiated approach to medicine phonophoresis in complex treatment of cicatricial deformations. Vestn Ross Akad Med Nauk. 2005;6:29.

13. Juckett G, Hartman-Adams H. Management of keloids and hypertrophic scars. Am Fam Physician. 2009;80(3): 253-260.

14. Wu WS, Wang FS, Yang KD, Huang CC, Kuo YR. Dexamethasone induction of keloid regression through effective suppression of VEGF expression and keloid fibroblast proliferation. J Invest Dermatol. 2006;126(6):1264-1271; doi: 10.1038/sj.jid.5700274.

15. Lee KL, Zhou Y. Quantitative evaluation of sonophoresis efficiency and its dependence on sonication parameters and particle size. J Ultrasound Med. 2015;34(3):519-526; doi: 10.7863/ultra.34.3.519.

16. Park D, Park H, Seo J, Lee S. Sonophoresis in transdermal drug delivery. Ultrasonics. 2014;54(1):56-65; doi: 10.1016/j.ultras.2013.07.007.

17. Van der Wal MB, van Zuijlen PP, van de Ven P, Middelkoop E. Topical silicone gel versus placebo in promoting the maturation of burn scars: a randomized controlled trial. Plast Reconstr Surg. 2010;126(2):524-531; doi: 10.1097/PRS.0b013e3181e09559.

18. Karagoz H, Yuksel F, Ulkur E, Evinc R. Comparison of efficacy of silicone gel, silicone gel sheeting, and topical onion extract including heparin and allantoin for the treatment of postburn hypertrophic scars. Burns. 2009; 35(8):1097-1103; doi: 10.1016/j.burns.2009.06.206.

19. Danielsen PL, Ru W, Ågren MS, Duke JM, Wood F, Zeng XX, et al. Radiotherapy and corticosteroids for preventing and treating keloid scars. Cochrane Database Syst Rev. 2013;12:CD010883; doi: 10.1002/14651858. CD010883.

20. Lee DW, Ku SK, Cho HJ, Kim JH, Hiep TT, Han SD, et al. Effects of Noscarna ${ }^{\mathrm{TM}}$ on hypertrophic scarring in the rabbit ear model: histopathological aspects. Arch Pharm Res. 2012;35(11):1999-2006; doi: 10.1007/s12272-0121117-4.

21. Wananukul S, Chatpreodprai S, Peongsujarit D, Lertsapcharoen P. A prospective placebo-controlled study on the efficacy of onion extract in silicone derivative gel for the prevention of hypertrophic scar and keloid in median sternotomy wound in pediatric patients. J Med Assoc Thai. 2013;96(11):1428-1433.

22. Chanprapaph K, Tanrattanakorn S, Wattanakrai P, Wongkitisophon P, Vachiramon V. Effectiveness of onion extract gel on surgical scars in Asians. Dermatol Res Pract. 2012;2012:212945; doi: 10.1155/2012/212945.

23. Ho WS, Ying SY, Chan PC, Chan HH. Use of onion extract, heparin, allantoin gel in prevention of scarring in Chinese patients having laser removal of tattoos: a prospective randomized controlled trial. Dermatol Surg. 2006;32(7):891-896; doi: 10.1111/j.1524-4725.2006. 32192.x.

24. Koc E, Arca E, Surucu B, Kurumlu Z. An open, randomized, controlled, comparative study of the combined effect of intralesional triamcinolone acetonide and onion extract gel and intralesional triamcinolone acetonide alone in the treatment of hypertrophic scars and keloids. Dermatol Surg.2008;34(11):1507-1514; doi:10.1111/j.1524-4725. 2008.34314.x. 